\title{
An Automatic Weight Control for Worsted on Railway-balling Head
}

\author{
By Fumio Murakami and Kanji Hosotsuji, Members, TMSJ \\ Toyo Spinning Co., Ltd.
}

\begin{abstract}
When an automatic weigh feeder is used on a worsted card, the discountinuous operation of the weigh feeder unavoidably causes periodic weight variations in the card sliver. While slivers from a number of cards are combined on the railwayballing head, the periodic weight variations in the individual card slivers are superimposed on the combined slivers and create periodic weight variations, with irregularly changing amplitudes. A slow weight variation due to a change in wool moisture also shows on the combined slivers.

The authors have designed an automatic sliver weight control device based on the open-loop control principle and used it on the railway-balling head to reduce weight variations in combined slivers. The control device has been found to eliminate periodic weight variations almost entirely, provided their wave length is more than 2 meters in terms of card slivers.
\end{abstract}

\section{Weight Variations in Combined Slivers}

The automatic weigh feeder deposits a fixed quantity of scoured wool at regular time intervals on the feed apron of a worsted card. The authors suspected that the intermittent deposition of wool and changes in wool moiture were responsible for most of the weight variations in combined silvers delivered by the railway-balling head. A series of experiments were made to investigate the first of the two suspected causes.

1-1. Measuring the weight variations in sliver delivered by balling head

Sliver balls delivered by the balling head were cut into consecutive 1-meter samples for a continuous weight measurement. The results for three of the balls are shown in Figures 1,2 and 3. Fig. ure 1 shows a ball with the largest C.V. $\%$ and Figure 3 one with the smallest C.V. \%. The 1-meter samples were spaced 25 centimeters apart to insure the correctness of the sample length.

Figures 1,2 and 3 show that all of the balls weighed had sinusoidal weight variations with varying amplitudes. Note also that the three balls shown in Figures 1,2 and 3 were not produced in succession but some time apart. This made it neccessary to gramms/meter

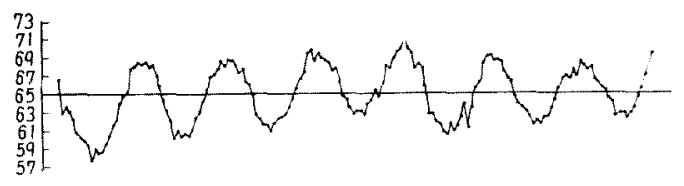

$\ddot{\mathrm{X}}=64.8 \mathrm{gramms} / \mathrm{meter}$

c.v. $=4.7 \%$

Fig. 1 Weight of sliver delivered by the railway-balling head without the weight control (consecutive one-meter cuts).

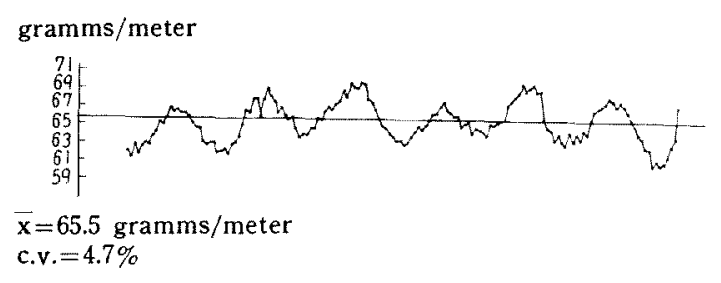

Fig. 2 Weight of sliver delivered by the railway-balling head without weight control (consecutive one-meter cuts).

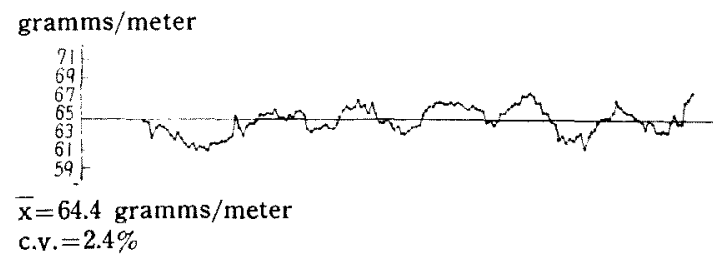

Fig. 3 Weight of sliver delivered by the railway-balling head without the weight control (cosecutive one-meter cuts). 
recheck the sinusoidal weight variations for a longterm change in their amplitude.

1-2. Long-term change in the amplitude of sinusoidal weight variation

Since it would be an extremely time-consuming job to cut many balls of slivers into consecutive 1 meter samples for measuring long-term weight variations, a short-cut was used: continuous measurement of the thickness of the combined slivers at the rear end of the railway-balling head.[i]

Figures 4 and 5 show the results of a test of about $21 / 2$ hours' duration. Figure 4 is part of the actual strip-chart record obtained. Figure 5 shows the thickness variations for the $21 / 2$-hour period condensed from the chart record. (Since it takes about 4 minutes to produce one ball, 37 balls equalling 7,400 meters of sliver were measured for thickness in $21 / 2$ hours.)

These long-term tests showed that slivers enter. ing the railway-balling head had a sinusoidal change in thickness where frequency was practically constant but where the amplitude fluctuated slowly.

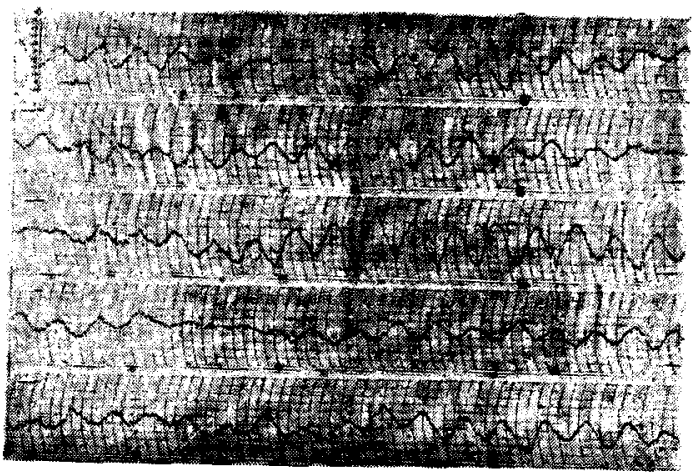

Fig. 4 Thickness of sliver entering the railway-baliing head (recorded by measuring roll)

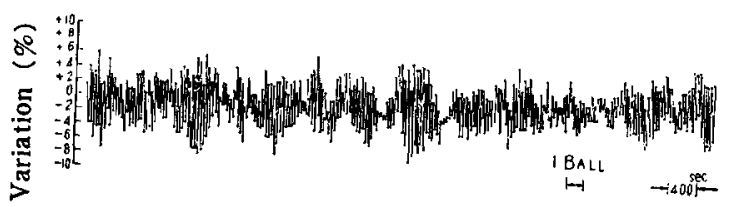

Fig. 5 Thickness variation of sliver entering the railway. balling head (recorded by measuring roll)

[i] The automatic sliver weight control device developed by the authors for the present experiment used a measuring roll at the rear end of the balling head to convert sliver thickness into a roll displacement (Figure 7). The roll displacement was electrically amplified and recorded on a strip chart to obtain long-term thickness variations.

\section{1-3. Frequency of sliver weight variations}

To determine the frequency of weight variations accurately, the data shown in Figure 1 was used to calculated the autocorrelation for the weight variations of the output sliver (sliver delivered by the balling head). The autocorrelation thus obtained is shown as curve A in Figure 6. Here the weight variations of the output sliver, rather than the input sliver, of the balling head were used to calculate the autocorrelation, for two reasons.

One reason was that effect of the automatic weight control could be readily evaluated in terms of the autocorrelation of the output sliver. The second reason was that the wave length of the weight variations of the input sliver could be readily converted into that of the output sliver by merely multiplying the draft ratio of the railway-balling head, which, in this case, was 1.5 .

Figure 6 shows that the sliver delivered by the balling head had an approximately sinusoidal weight variation 30.6 meters in wave length. (The draft ratio of the balling head being 1.5, 30.6 meters of the output sliver was equivallent to 20.4 meters of input sliver.) Curve B in Figure 6 is a true sinusoid with an amplitude of 7.2 (gramms/meters) ${ }^{2}$ and a wave length of 30.6 meters. The high proximity of curve $A$ to curve $B$ indicates that the abovementioned sinusoid is the prevailing component of the weight variations shown in Figure 1.

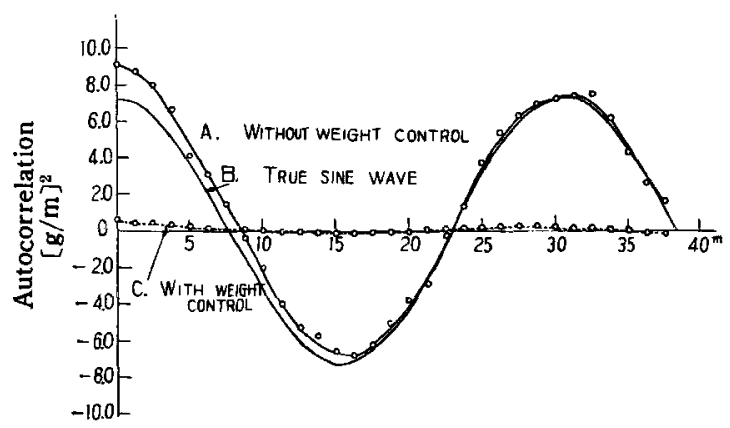

Fig. 6 Autocorrelation functions for slivers delivered by the railway-balling head with and without the weight control

[ii] Wave length of the weight variation in the input sliver ........... 20.4 meters

Surface speed at the back roll of the balling head 0.52 meters $/ \mathrm{sec}$

Period of the weight variation in the input sliver $20.4 / 0.52=39.2$ seconds

Deposition interval of the automatic weigh feeder 38 seconds 
It was found that this wave length, when converted into frequency per minute, was approximately equal to the number of times per minute[ii] the automatic-weigh feeder deposited wool on the feed apron of the card.

The authors had long been aware that the automatic weigh feeder which deposited wool in a transverse direction on the feed lattice caused some periodic weight variations in the card sliver.

However, the authors expected that such pariodic weight variations in the card sliver would be practically eliminated by the doubling of slivers in the railway-balling head. Yet, a considerable weight variation with a frequency approximately equal to that of the automatic weigh feeder was found to exist in a combined sliver delivered by the balling head.

\section{1-4. Amplitude of weight variation}

A theoretical interpretation is given here to the fluctuations in the amplitude of periodic weight variations. Let us assume that the weight of a card sliver is a time function and, as such, can be approximated with the function

$$
x+a \sin (w t+\varphi)
$$

where: $x$ is the average sliver weight per meter; $a$ is the amplitude of the sinusoidal weight variation; $w$ is the angular frequency of the sinusoidal weight variation (approximatery equal to the angular frequency of the automatic weigh feeder):

$t$ is time; and $\varphi$ is the phase angle.

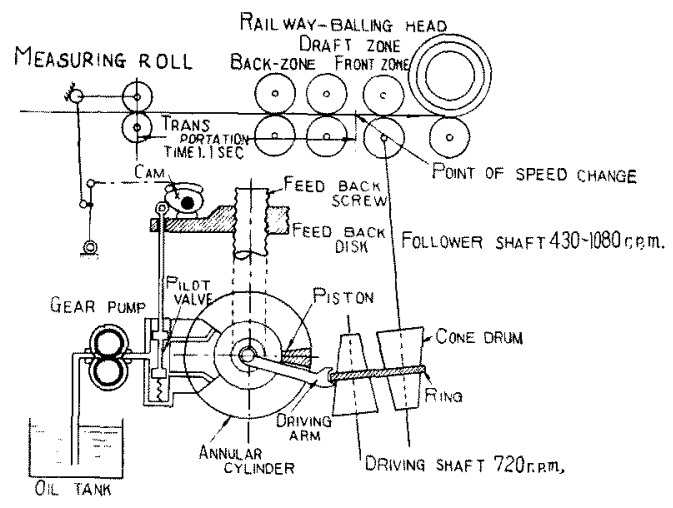

Fig. 7 Automatic weight control device

[iii] Subscript $i$ is used on the angular frequency $w_{i}$, be cause each weigh feeder has a slightly differing operating frequency due to belt slippage and other reasons. The range of the deposition interval $2 \pi / w_{l}$ was found to be 37.8 $\sim 38.2 \mathrm{sec}$.
When $n$ of such card slivers are combined at the railway-balling head, the weight of the combined slivers is

$$
\sum_{i=1}^{n_{b}} X_{t}+\sum_{i=1}^{n} a_{i} \sin \left(w_{\ell} t+\varphi_{i}\right)^{[\mathrm{iii}]}
$$

Here the phase angle $p_{t}$ should be understood to include the phase shift arising from the difference in time required to transport silvers from individual cards to the balling head.

The first term of equation (2) is the average weight of the combined slivers; the second term $\sum_{t=1}^{n} a_{i} \sin \left(w_{i} t+\varphi_{i}\right) \quad$ is a variable of time.

For the sake of simplicity, let us also assume arbitrarily that

$$
a_{\ell}=1
$$

The second term, which we call $X$, then transforms itself into:

$$
X=\sum_{i=1}^{n} \sin \left(w_{i} t+\varphi_{i}\right)
$$

where the values of $w_{i}$ are all close to one another, as stated before.

The sum in equation (4) cannot be expressed in a simple closed form when $n$ exceeds 2 . How. ever, the values of $w_{i}$ being close to one another, we divide $w_{i}$ into two parts: the average $w_{0}$ and the deviation $\Delta w_{i}$ from the average.

$$
w_{i}=w_{0}+\Delta w_{i} \quad \Delta w_{i} \ll w_{0}
$$

Substituting (5) into (4) gives:

$$
\begin{aligned}
X & =\sum_{i=1}^{n} \sin \left(w_{t}+\varphi_{i}\right) \\
& =\sum_{i=1}^{n} \sin \left(w_{0} t+\Delta w_{i} t+\varphi_{i}\right) \\
& =\sum_{i=1}^{n} \sin \left(w_{0} t+\varphi_{i}^{\prime}\right)
\end{aligned}
$$

where

$$
\varphi_{i}^{\prime}=\Delta w_{i} t+\rho_{t}
$$

The coefficient of $t$ now being a constant $w_{0}$, equation (6) can be rewritten in this form:

$$
\begin{aligned}
X & =\sum_{i=1}^{n} \sin \left(w_{0} t+\varphi_{i}^{\prime}\right) \\
& =\sin \left(w_{0} t+\emptyset\right)
\end{aligned}
$$

where

$$
\begin{aligned}
& A^{2}=n+2\left[\cos \left(\varphi_{1}^{\prime}-\varphi_{2}^{\prime}\right)+\cdots \cdots+\cos \left(\varphi_{1}^{\prime}-\varphi_{n}{ }^{\prime}\right)\right. \\
& +\cos \left(\varphi_{z}^{\prime}-\varphi_{3}{ }^{\prime}\right)+\quad+\cos \left(\varphi^{\prime}-\varphi_{n}{ }^{\prime}\right) \\
& \left.+\cos \left({\varphi^{\prime}}_{n-1}^{\prime}-\varphi_{n^{\prime}}\right)+\cdots \cdots+\cos \left(\varphi_{p^{\prime}}^{\prime}-\varphi_{n}{ }^{\prime}\right)\right] .
\end{aligned}
$$


and

$$
\begin{aligned}
& \Phi=\tan ^{-1}\left[\left(\sin \varphi_{1}^{\prime}+\sin \varphi_{z}^{\prime}+\cdots \cdots \cdots+\sin \varphi_{n^{\prime}}\right)\right\} \\
& \left.\left(\cos \phi_{1}{ }^{\prime}+\cos p_{2}{ }^{\prime}+\cdots \cdots \cdots+\cos \phi_{n}{ }^{\prime}\right)\right) \cdots \cdots(9)
\end{aligned}
$$

Both $A$ and $\Phi$ are functions of time, but their rate of change with respect to time is much smaller than $w_{0} t$, since time $t$ always appears in $A$ and $t$ in the form of $\Delta w_{i} t$ where the coefficient $\Delta w_{i}$ is much smaller than $w_{0}$. It follows, then, that the weight variation in a combined sliver as shown in equation (7) is a modulated sine wave whose frequency is approximately equal to the operating frequency of the automatic weigh feeder and whose amplitude fluctuates only slowly with time. This agrees with the experimental data given in Figures 4 and 5.

\section{Automatic Sliver Weight Control Device}

To attenuate the weight variation in the combined slivers which was found approximately to be a modulated sine wave, the authors developed an automatic weight control device based on the openloop control principle.

\section{2-1. Configuration of the control device}

The automatic weight control device the authors developed, like the Autoleveler, is based on the open. loop control principle. In other words, there is no feedback of information involved in cotrolling the sliver weight. A measuring roll at the rear end of the balling head converts input sliver thickness into a roll displacement, and a hydraulic servo drives a variable speed device to change the front roll speed of the balling head in response to the roll displacement.

Since the dominent weight variation to be atten. uated is of a long wave length, 30.6 meters, no timedelay instrument is used in the weight control device. This makes the control device considerably lower in cost than similar commercial machines, such as the Autoleveler.

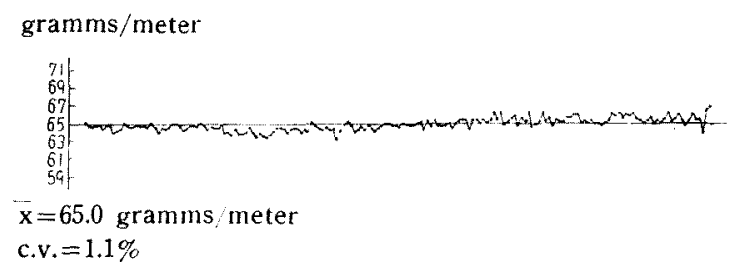

Fig. 8 Weight of sliver delivered by the railway-balling head with automatic weight control (consecutive one-meter cuts)
The variable speed device used here consists of a pair of cone drums engaged to a ring, the position of the ring being shifted sidewise by means of a hydraulic servo. The variable speed device is illustrated in Figure 7.

\section{2-2. Test results}

(a) Sliver weight variation within a ball

Slivers produced by the railway-balling head equipped with the weight control device were cut into consecutive 1-meter samples for weighing in the same manner as already described. The results of the weighing are given in Figure 8 . A comparison of Figure 8 with Figures 1,2 and 3 shows clearly that the weight control device eliminates most of the periodic weight variations in the output sliver. Curve $C$ in Figure 6 shows the autocorrelation function for the weight variation derived from the data shown in Figure 8.

A comparison of curve $C$ with curve $A$ in Figure 6 again shows that weight variations are almost entirely eliminated by the control device. The eff. cacy of the weight control is also proved by the C.V. versus $L$ curves in Figure 9.

(b) Ball weight variation

To determine how the weight control affected long-term weight variations, balls produced with and without the weight control were weighed suc. cessively as shown in Figures 10 and 11 , each ball

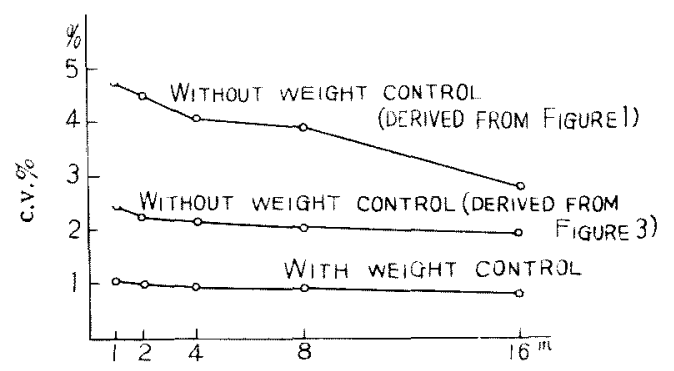

Fig. 9 Sample length versus C. V.

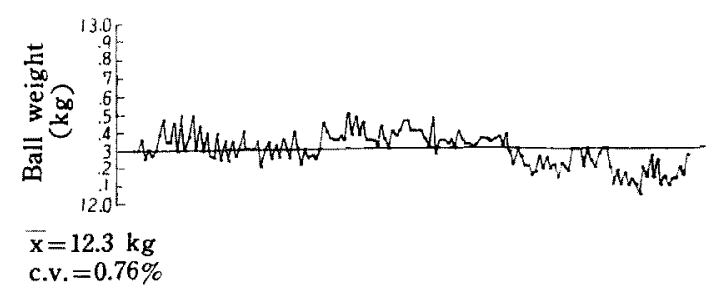

Fig. 10 Ball weight variations in railway-balling head equipped with automatic weight control device 
containing an approximately equal length of sliver. Figures 10 and 11 shows that the coefficient of variations was reduced by about $30 \%$ by the weight control.

(c) Effect of wool moisture on ball weight variations

The automatic-weigh feeder weighs scoured wool without adjustments for wool moisture. Therefore, any large variation in the moisture content of wool entering a card results in a corresponding weight variation in the card sliver, because carding has the effect of evening out moisture variations making the moisture content in the card sliver almost constant.

Accordingly, it was expected that the effect of moisture variations was the principal cause of the long-term weight variations present in the slivers produced without the aid of the weight control (Figure 10). A statistical study confirmed that a correlation existed between the ball weight and wool moisture for a risk of $5 \%$ (Figure 12).

It is clear, then, that most of the long-term weight variations shown in Figure 10 were attributable to moisture variations and could have been eliminated if the wool had been given uniform moisture. In other words, when wool moisture is reasonably controlled, the measuring roll of the weight control device can be considered to measure sliver weight with enough accuracy for weight control.

2-3. Mathematical model for the weight control device

The operation of the sliver weight control device can be approximated by a simple mathematical model derived from the following assumptions:

1. That no change in sliver weight occurs while a sliver is transported from the measuring roll to the draft zone.

2. That the travelling speed of fibers within a sliver suddenly changes from the back roll speed to the front roll speed at a fixed point between the two rolls, that point being called the point of speed change.

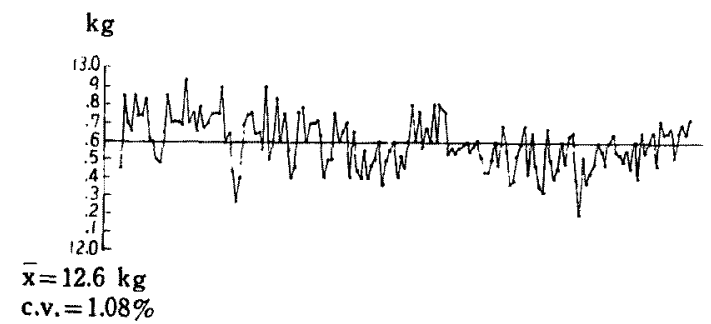

Fig. 11 Weight variations in balls delivered by the railwayballing head without weight control
3. That drafting operation is approximated by the division of the input sliver weight by the draft ratio which is the ratio of the front roll surface speed to the back roll surface speed.

4. That no change in sliver weight occurs while the slivers are being wound into balls.

A block diagram describing the weight control device based on these assumptions is shown in Figure 13. The notations used in the block diagram are:

$z$ input sliver weight per meter;

$z_{0}$ average value of $z$;

$z_{1}$ deviation from the average $z_{0}$ as a time function;

$x$ output sliver weight per meter;

$\mathrm{x}_{0}$ average value of $\mathrm{x}$;

$\mathrm{x}_{1}$ deviation from the average $\mathrm{x}_{0}$ as a time function ;

$\mathrm{d}_{0}$ average draft ratio;

$d_{1}$ deviation from the average $d_{0}$ as a time function;

$\mathrm{L}_{f}$ time required to transport slivers from the measuring roll to the point of speed change;

$\mathrm{K}$ proportional gain of the control device;

$\mathrm{T}$ time constant of the variable speed device.

It is assumed here that the characteristics of the variable speed device is approximated by a firstorder lag.

The numerical values of $\mathrm{L}_{f}, \mathrm{~K}$ and $\mathrm{T}$ are substituted in Figure 13 to obtain Figure 14 . Here $\mathrm{L}_{f}$ is calculated on the following assumptions:

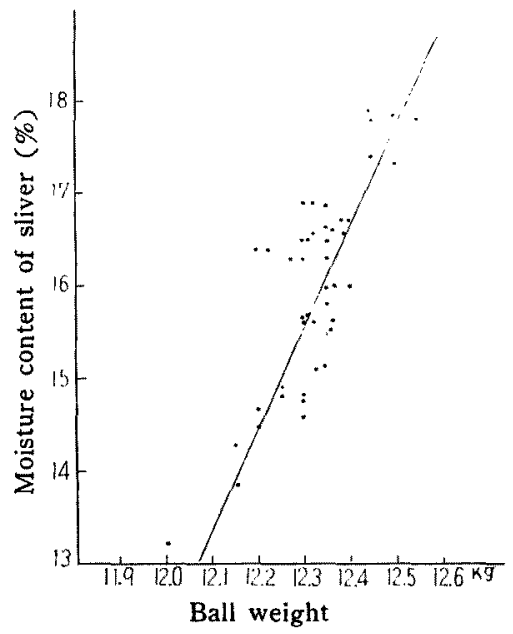

Fig. 12 Relation between ball weight and moisture content (when the automatic sliver weight control device is not used) 
1. That the speed of a sliver is equal to the surface speed of the roll in contact.

2. That the point of speed change is located at a point half the mean fiber length back of the front roll.

3. That the mean fiber length is 66 milimeters.

The time constant $T$ was obtained experimentally by measuring the front roll speed in response to a sudden movement in the measuring roll using a recording tachometer.

The reducing ratio $A(u)$, then, is derived for the block diagram in Figure 13 by the procedure described in a previous report by the same authors (Figure 15).[iv]

Figure 15 shows that the function $A(u)$ is less than 1 for values of $(u)$ less than 1 . In other words, the control device is theoretically capable of attenuating sliver weight variations in an input sliver of a wave length exceeding 2 meters. As stated earlier, the predominant weight variation in an uncontrolled input sliver is a sinusoid 20.4 meters in wave length.

Figure 15 also shows that the value of $\mathrm{A}(\mathrm{u})$ for this particular wave length is 0.1 . That is to say, the dominant part of the weight variation would be reduced by $90 \%$ by the control device. This agrees fairly well with the experimental results shown in Figures 10 and 11.

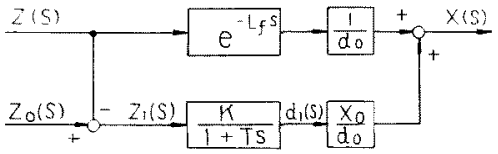

Fig. 13 Block diagram for the weight control device

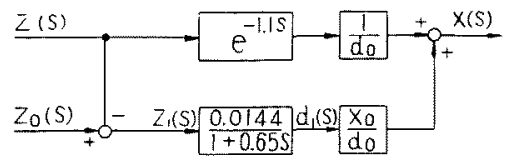

Fig. 14 Block diagram for the weight control device

[iv] Murakami, F., Shoman, T., and Kase, S., Journal of the Textile Machinery Society of Japan. Vol. 12, No. 4. The reducing ratio $A(u)$ of the weight control device is the ratio by which a sinusoidal weight variation of the output sliver is reduced by the control device. It is also a function of the normalized frequency of the weight variation. The shape of the $A(u)$ curve for the control device described by the block diagram in Figure 13 depends on the value of parameter $\gamma$. $\gamma$ value for this particular control device is obtained by substituting $L^{\prime}=0, L_{f}=1.1 \mathrm{sec}$ and $T_{a}=0.65 \mathrm{sec}$ into the following formula:

$$
\gamma=\left(\mathbf{L}^{\prime}-\mathbf{L}_{J}\right) / \mathrm{T}_{a}=-1.1 / 0.65=-2
$$

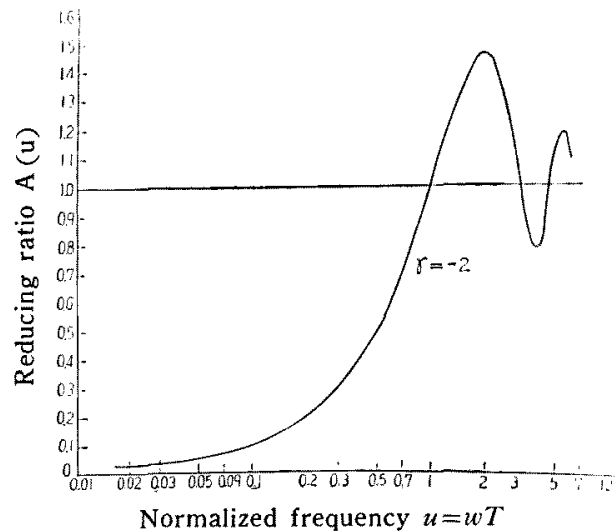

Fig. 15 Reducing ratio for the weight control device

\section{Conclusions}

The automatic weight control device developed by the authors for the railway-balling head was primarily intended to be an inexpensive instrument for attenuating long-term weight variations.

The device was shown by tests to eliminate almost entirelythose weight variations induced by:

(i) the intermittent operation of the automatic weigh feeder

(ii) the inaccuracy of automatic weighing arising from accidental shortage of wool in the feed hopper or from other causes

(iii) and variations in wool moisture (Even when the automatic-weigh feeder is correctly operating variations in wool moisture result in a corresponding variation in sliver weight as the moisture gets levelled out during carding.)

The authors express their deep appreciation to Prof. K. Nakajima of the Municipal College of Osaka for his valuable advice which has made this study possible. 